\title{
Sensitivity of Dirac fermion and Weyl fermion by the Energy Conservation Law of Lenz
}

Teresa Oh ( $\square$ teresa@cju.ac.kr)

cheongju university https://orcid.org/0000-0002-4194-381X

Xiangjiang Li

School of Information Engineering, Nanyang Institute of Technology

Article

Keywords: Law of Lenz, Dirac fermion, Weyl fermion

Posted Date: December 1st, 2020

DOI: https://doi.org/10.21203/rs.3.rs-111834/v1

License: (c) (i) This work is licensed under a Creative Commons Attribution 4.0 International License. Read Full License 


\section{Abstract}

The law of Lenz as an energy conservation law between the electric field and the magnetic field allows the charge current and spin current generated by matters and antimatters to be symmetrical with each other. Thus, there is a Lenz plane of symmetry to the electromagnet energy. Phase isolators are charged current by electrons and spin current by spin. The charge current is Weyl fermion, and the spin current is Dirac fermion. The charge current and spin current are characterized by balancing each other, and the fermion, which has both particles and carriers, is Majorana fermion. The reaction was investigated when phase insulators in the state of Dirac fermion and Weyl fermion were exposed to $\mathrm{CO} 2 \mathrm{gas}$. Although the sensitivity of the phase isolator has decreased as Weyl fermion makes spin current in the gas environment, the sensitivity of the phase isolator has increased as Dirac fermion makes charge current in the gas environment. The spin current of the Dirac fermion has a super current characteristic with a resistance of zero and quantum tunneling phenomenon has occurred. According to Lenz's energy conservation law, the electron sensor has an advantage of the phase insulator in the state of Dirac fermion, where the charge current increases.

\section{Introduction}

The study of superconductors operating on the basis of magnetic energy is mainly dealt with in the field of spintronics and is classified as a twoclass superconductor. The study of superconductors began in 1911 when mercury was observed to be resistance zero by the Onnes of the Netherlands, and the principle of operation of superconductors was found to be BCS theory. In 1957, the BCS theory explained the superconductor theoretically with two pairs of electronic coopers consisting of reverse spin direction. In order to confirm superconductivity, the resistance must be demonstrated to be zero, to prevent the moving of electrons and to appear the moving of spins, Meissner effect (resistance $\mathrm{R}=0$ ) occurs in a strong magnetic field and at very low temperatures. ${ }^{1,2}$ In superconductors, there are many materials that have emerged as semiconductor technology has developed. The theory of copper-based semi-conductors in 1987 and iron-based superconductors in 2006 is described as spin-density wave (SDW). A superconductor that has been studied recently is a phase insulator. Phase insulators are internal insulators, but surface currents flow from the surface. To become a topological insulator, a small, thin 2D structure and magnetic field and low temperature are required. ${ }^{3-5}$

By using semiconductor-specific process technology, it can be made from a large area of material. The same material also increases its energy electromagnetic as its surface area widens. Thus, the topological insulator has superconductivity without magnetic field due to its structural effect with a large area.

Therefore, topological insulators have high surface energy, so the electronic energy relationships and differences of common insulators appear. Typically, topological insulators exhibit superconductivity without a magnetic field. This is called the Anomalous Quantum Hall Effect (AQHE). The surface current of the phase insulator starts from the kinetic energy of the spin rotation by the kinetic momentum of the electron. The magnetic field is generated by the electro-momentum and spin motion, and magnetic energy forms a spin current. ${ }^{6-8}$ The spin current is the charge surface current at the surface. By the Maxwell equation and the Stock's law, the topological insulator is an insulator, but a surface current is generated. The spin current is not a leakage current, but a super current. Normal insulators become leakage currents because spin currents cannot occur.

The electron and spin are related to the matter and the antimatter. The electron, which is the matter, makes the charge current, and the spin, which is the antimatter, makes the spin current. ${ }^{9-12}$ Matters and antimatters have the same physical quantity physically and chemically, but have a relationship of chiral symmetry, with opposite polarity electrically. Therefore, the charge current and spin current are opposite in polarity. The charge current has a + resistance in the field, and the spin current shows a negative resistance in the magnetic field. The spin current of the topological insulator is negative resistance characteristic and there is no loss of thermal resistance, so the super current flows. ${ }^{13-16}$ Thus the topological insulator is an insulator, but it is the principle of current flow. Studies of insulators have led to studies of schottky contact and low-k materials, ${ }^{17-19}$ and recently reported that low-k materials are phase insulators. ${ }^{3}$

There has been a lot of research in the past on the cause of the increase in current values due to schottky contact. Schottky contact is caused by a depletion layer that occurs in the interface of semiconductor PN junction, and it is a good environment for schottky contact to make spin current, so it is highly likely that super current will be created. ${ }^{20-21}$ Schottky resistance by schottky contact is the opposite concept of ohmic resistance. Low-k material produces spin current from a material that implements a schottky contact, so low-k makes it 10 times faster than normal OTFT movement. 22

The matters and antimatters that make up the phase insulator include Majorana fermion, Dirac fermion and Weyl fermion. The Weyl fermion appears in conductive materials, and the Majorana fermion and Dirac fermion, which appear in insulating materials, have characteristics that are so common that it is hard to distinguish.

Since electronic energy is preserved from the kinetic energy of electrons and spins, the energy conservation laws of matters and antimatters are preserved. Matters and antimatters have a symmetrical plane. ${ }^{23-24}$

It is due to the law of Lenz that the energy of the electric field and the magnetic field can be preserved. For Lenz's energy conservation law to be possible, there is a symmetric plane in which energy is converted in the symmetry relationship between electrons and spins. Electrical symmetry 
plane is positive and negative resistance, and is a function of temperature. Electric fields are positive resistance areas with charge currents, and magnetic fields are negative resistance areas with spin currents. Tesla's cold electricity works in the negative field by energy conservation law of Lenz. ${ }^{25}$ In the equation of Dirac, the negative resistance region is like the sea of Dirac, which means magnetic energy, and has two-dimensional wave energy.

In this study, it was studied the principle of making topological insulator and the negative resistance characteristic from the generation of spin current and the supercurrent being made.

\section{Experimental Processes}

A p-type silicon wafer with $\mathrm{SiO}_{2}$ was used as a substrate, and $\mathrm{SiO}_{2}$ thin film was deposited by magnetron sputtering method, supplying 25 sccm and $30 \mathrm{sccm}$ of argon gas on top of it. After deposition of $\mathrm{SiO}_{2}$ thin film, ZTO was treated for 10 minutes with Ar=20 sccm, and the effect of varying conductivity was investigated as the electrical characteristics of $\mathrm{ZTO} / \mathrm{SiO}_{2}$ thin. And the correlation between conductivity and the depletion layer formed in the semiconductor junction was studied. The change in charge amount was investigated by measuring capacitance, and the currentvoltage characteristics were investigated to compare and analyze the correlation between capacitance and spin current. To examine the contact

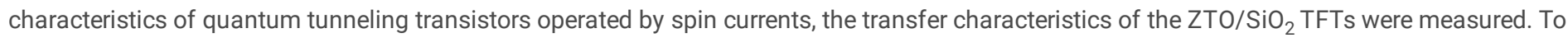
researched the difference between Majorana fermion and Dirac fermion, the quantum tunneling phenomenon of transistors owing to the reaction between matters and antimatters was measured.

\section{Results And Discussion}

Matters and antimatters have electro-magnetic energy composed of electric field and magnetic field by electron matters and spin antimatters. In Figure 1, matters are three-dimensional structures, and the antimatters are obtained from two-dimensional structures. The study of matters and antimatters has developed into classical mechanics that study matter and quantum mechanics that study the state of matter. The energy conservation law is maintained because it has the same energy value although the three dimensional kinetic energy ( $E=m c 2)$ and two dimensional wave energy $(E=h y)$. As the dimension changes, the plane of symmetry changes. In accordance with the Lenz Act, which is the energy conservation law of the electric field and the magnetic field, the Lenz plane, which becomes the reference plane where the electric field and the magnetic energy are symmetrical, is the threshold voltage in three dimensions. It is Weyl fermion that the energy moves in one direction according to the threshold voltage. Dirac fermion and Majorana fermion are energy state that follow the Dirac function, which are treated in two-dimensional quantum mechanics. The two-dimensional Dirac function is symmetrical, and the criterion of symmetry is Fermi-level. Electrically, if the fermi-level and the Lenz plane are in the same position, the energy is in the Majorana fermion state. However, it is difficult to make in the case of Majorana fermion, and most of the energy is in the state of Dirac fermion or Weyl fermion.

Matters and antimatters have electro-magnetic energy composed of electric field and magnetic field by electron matters and spin antimatters. In Figure 1, matters are three-dimensional structures, and the antimatters are obtained from two-dimensional structures. The study of matters and antimatters has developed into classical mechanics that study matter and quantum mechanics that study the state of matter. The energy conservation law is maintained because it has the same energy value although the three dimensional kinetic energy ( $E=m c 2)$ and two dimensional wave energy $(E=h y)$. As the dimension changes, the plane of symmetry changes. In accordance with the Lenz Act, which is the energy conservation law of the electric field and the magnetic field, the Lenz plane, which becomes the reference plane where the electric field and the magnetic energy are symmetrical, is the threshold voltage in three dimensions. It is Weyl fermion that the energy moves in one direction according to the threshold voltage. Dirac fermion and Majorana fermion are energy state that follow the Dirac function, which are treated in two-dimensional quantum mechanics. The two-dimensional Dirac function is symmetrical, and the criterion of symmetry is Fermi-level. Electrically, if the fermi-level and the Lenz plane are in the same position, the energy is in the Majorana fermion state. However, it is difficult to make in the case of Majorana fermion, and most of the energy is in the state of Dirac fermion or Weyl fermion.

The depletion layer is caused by two PN junctions, and the depletion layer creates a schottky contact. As shown in Figure 3 , the schottky contact is caused by magnetic field energy generated by the potential barrier. The spin current produced by magnetic energy is two-dimensional structure, including both positive (+) directional current and negative (-) directional current.

Figure 4 illustrates the formation of the schottky contact and spin current. SiO2 insulation film is made, which shows a difference in the characteristics of the current. Figure 4(a) also shows a difference in capacitance. The capacitance value of SiO2 (Ar=25 sccm) insulating film with high current flow is also high. Figure 4(b) shows that the schottky contact was formed on SiO2 (Ar=25 sccm) insulation film, but the spin current is flowing on the $\mathrm{SiO} 2(\mathrm{Ar}=30 \mathrm{sccm})$ insulation film.

Figure 5 shows the transmission characteristics of transistors. Transistors with $\mathrm{SiO} 2$ ( $\mathrm{Ar}=25 \mathrm{sccm})$ insulation film as gate insulation film have a high positive current, but transistors with $\mathrm{SiO} 2(\mathrm{Ar}=30 \mathrm{sccm})$ insulation film have a high negative current. In Figure 4(b), the TFT25 transistor showing the schottky contact is Weyl fermion and shows that the threshold voltage is moving as shown in figure 5(a). And it can be seen that the TFT30 transistor, where tunneling phenomena occur, is in the state of Dirac fermion as shown in figure 5(b). 
The depletion layer is caused by two PN junctions, and the depletion layer creates a schottky contact. As shown in Figure 3, the schottky contact is caused by magnetic field energy generated by the potential barrier. The spin current produced by magnetic energy is two-dimensional structure, including both positive $(+)$ directional current and negative $(-)$ directional current.

Figure 4 illustrates the formation of the schottky contact and spin current. SiO2 insulation film is made, which shows a difference in the characteristics of the current. Figure 4(a) also shows a difference in capacitance. The capacitance value of SiO2 (Ar=25 sccm) insulating film with high current flow is also high. Figure 4(b) shows that the schottky contact was formed on $\mathrm{SiO} 2$ ( $\mathrm{Ar}=25 \mathrm{sccm})$ insulation film, but the spin current is flowing on the $\mathrm{SiO} 2(\mathrm{Ar}=30 \mathrm{sccm})$ insulation film.

Figure 5 shows the transmission characteristics of transistors. Transistors with $\mathrm{SiO} 2$ ( $\mathrm{Ar}=25 \mathrm{sccm}$ ) insulation film as gate insulation film have a high positive current, but transistors with $\mathrm{SiO} 2(\mathrm{Ar}=30 \mathrm{sccm})$ insulation film have a high negative current. In Figure 4(b), the TFT25 transistor showing the schottky contact is Weyl fermion and shows that the threshold voltage is moving as shown in figure 5(a). And it can be seen that the TFT30 transistor, where tunneling phenomena occur, is in the state of Dirac fermion as shown in figure 5(b).

Quantum tunneling phenomena are related to sensitivity. The current characteristics were investigated by injecting 100 sccm of $\mathrm{CO} 2 \mathrm{gas}$ from Weyl fermion and Dirac fermion.

Figures $7(\mathrm{a})$ and Figure $7(\mathrm{~d})$ are the result of $\mathrm{CO}_{2}$ gas reaction to $\mathrm{TFT}_{25}$ transistor, and the charge current in $\mathrm{TFT}_{25}$ is increasing in the environment with gas. Figures $7(\mathrm{~b})$ and 7 (c) are transfer characteristics of $\mathrm{I}_{\mathrm{DS}^{-}} \mathrm{V}_{\mathrm{GS}}$ in $\mathrm{TFT}_{30}$ and figures $7(\mathrm{e})$ and $7(\mathrm{f})$ are characteristics of $\mathrm{I}_{\mathrm{DS}}-\mathrm{V}_{\mathrm{GS}}$ transfer in $\mathrm{TFT}_{30}$ in an environment with gas. Spin current shows a rapid increase in charge current in an environment with gas. Based on the Lenz plane, the charge current in the Weyl fermion state is moving in the direction of spin current in the gas environment according to the energy conservation law of the electric field and the magnetic field. The spin current in a relatively Dirac fermion state is moving in the direction of the charge current in the gas environment. If the environment changes due to gas, it can be seen that electronic energy is changing as the charge current and spin current are balanced according to Lenz's energy conservation law.

Figure 8 investigated the phenomenon of changing current by converting the results of Figure 7 into logarithmic values. Figures $8(a)$ and Fig. 8(b) show in $\mathrm{TFT}_{25}$ that the charge current is shifted to the spin current in the gas environment. In the state of Weyl fermion, the current of charge is decreasing as the current of charge is created to match the energy of the electric field and the magnetic field by Lenz's law. Thus, $\mathrm{CO}_{2}$ gas reactivity was reduced. However, figure 8(c) and figure 8(d) show that even in the gas environment, the spin current has changed little, and the charge current has increased significantly. Since the Dirac fermion's state is an area where supercurrent flows because the Lenz plane of the electric field and the magnetic field is identical, it can be seen that the spin current greatly increases the charge current above the threshold voltage as shown in Figure 4.

Spin and electrons are sources of making magnetic and electric fields, and according to Lenz's law, energy conservation laws are applied. Electron devices with high spin currents by applying the Dirac fermion state can be found to be advantageous for high-sensitivity electronic sensors because they generate a lot of charge currents when the environment changes.

\section{Conclusions}

The phase insulator using Renzi's energy conservation law has a state of Dirac fermion and a state of Weyl fermion. Majorana fermion is the intermediate state of Weyl fermion and Dirac fermion, indicating the energy state of matters and antimatters. Dirac fermion was observed to have supercurrent flow when the Weyl fermion was a schottky contact. The sensitivity in the gas environment was investigated by making Weyl fermion transistors and Dirac fermion transistors. The sensitivity in the gas environment was investigated by making a transistor of Weyl fermion and a transistor of Dirac fermion. As an energy conservation law in which the energy of electric field and magnetic field is symmetrical, the sensitivity of Weyl fermion transistor decreased as spin current was created in the gas environment according to Lenz's law. However, the Dirac fermion transistor has increased its sensitivity as it creates a charge current in a gas environment. Therefore, it can be seen that the phase insulator of Dirac fermion is advantageous to make a high-sensitivity sensor.

\section{Declarations}

\section{Author contributions}

T. O. conceived the study. All authors designed the experiment(s), and analyzed the results. All authors discussed the results and contributed to writing the manuscript.

Competing interests. All other authors have no conflict of interest to declare.

\section{References}

1. Yoshishige Suzuki, Hitoshi Kubota, Spin-Torque Diode Effect and Its Application. J Phys Soc Jpn 77, 031002 (2008). 
2. Philip F Bagwell. Suppression of the Josephson current through a narrow, mesoscopic, semiconductor channel by a single impurity. Phys Rev B, 46, 12573-86 (1992).

3. Wei Han, YoshiChika Otani and Sadamichi Maekawa, Quantum materials for spin and charge conversion,npj Quantum Materials, 27, 8308 (2018)

4. Giant Transition-State Quasiparticle Spin-Hall Effect in an Exchange-Spin-Split Superconductor Detected by Nonlocal Magnon Spin Transport, Kun-Rok Jeon, Jae-Chun Jeon, Xilin Zhou, Andrea Migliorini, Jiho Yoon, and Stuart S. P. Parkin, ACS Nano, 19, 139- 152 (2020).

5. Wei Han, Sadamichi Maekawaand Xin-Cheng Xie, Spin current as a probe of quantum materials, Nature Materials, 19, 139-152 (2020).

6. T. Oh, Analysis of Surface Current by Quantum Tunneling Effect of Thin Film Transistors with Topological Insulators, Scientific Reports, 10, 9509 (2020).

7. Rui Wang, Onur Erten, Baigeng Wang, D.Y. Xing, Prediction of a topological $p+$ ip excitonic insulator with parity anomaly. Nature Communications, 10, 210 (2019).

8. T. Oh. and Choi, C. K., Comparison between SiOC Thin Films Fabricated by Using Plasma Enhance Chemical Vapor Deposition and $\mathrm{SiO}_{2} \mathrm{Thin}$ Films by Using Fourier Transform Infrared Spectroscopy. J. Korean Phys. Soc. 56, 1150-1155 (2010).

9. Charles L. Kane, An insulator with a twist nature physics, CONDENSED MATTER, 4, 348-349 (2008).

10. Eric G Barbagiovanni, David J Lockwood Peter J Simpson, Lyudmila V Goncharova. Quantum confinement in Si and Ge nanostructures: Theory and experiment. Appl Phys Rev. 1, 011302 (2014).

11. T Edvinsson. Optical quantum confinement and photocatalytic properties in two-, one- and zero-dimensional nanostructures. Royal society open science 5, 180387 (2018).

12. J Frenkel. On pre-breakdown phenomena in insulators and electronic semi-conductors. Phys Rev54, 647-648 (1938).

13. Yuan Li, Yong Sun, Weiwei Zhu, Zhiwei Guo, Jun Jiang, Toshikaze Kariyado, Hong Che and Xiao Hu, Topological LC-circuits based on microstrips and observation of electromagnetic modes with orbital angular momentum, Nature Communications, 9, 4598 (2018).

14. Jungho Mun, Minkyung Kim, Younghwan Yang, Trevon Badloe, Jincheng Ni, Yang Chen, Cheng-Wei Qiu and Junsuk Rho, Mun et al. Electromagnetic chirality: from fundamentals to nontraditional chiroptical phenomena, Light: Science \& Applications, 9, 139 (2020).

15. P B Wiegmann. One-dimensional Fermi system and plane xy model. J Phys C Solid State Phys.11, 1583 (1978).

16. Raymond T Tung. The physics and chemistry of the Schottky barrier height. Appl Phys Rev. 1, 011304 (2014).

17. J Maserjian, N Zamani. Behavior of the $\mathrm{Si} / \mathrm{SiO}_{2}$ interface observed by Fowler Nordheim tunneling. Appl Phys Lett. 53, 559-567(1982).

18. Jeffrey $\mathrm{C} \mathrm{K}$, et al. Evidence of ultra-low-k dielectric material degradation and nanostructure alteration of the Cu/ultra-low-k interconnects in timedependent dielectric breakdown failure. Appl Phys Lett. 102,022908 (2013).

19. J. G. Simmon. Poole-Frenkel Effect and Schottky Effect in Metal-Insulator-Metal Systems.Phys Rev. 155, 657-660 (1967).

20. L L Chang, L Esaki, R Tsu. Resonant tunneling in semiconductor double barriers. Appl Phys Lett. 24, 593-595 (1974).

21. Harold P Hjalmarson, P Vogl, D J Wolford, John D Dow. Theory of Substitutional Deep Traps in Covalent Semiconductors. Phys Rev Lett. 44, 810-813 (1980).

22. T. Oh, Organic Thin-Film Transistors Using Pentacene and SiOC Film IEEE TRANSACTIONS ON NANOTECHNOLOGY, 5(1), 23 (2006).

23. N V Volkov, A S Tarasov, E V Eremin, F A Baron, S N Varnakov, S G Ovchinnikov. Extremely large magnetoresistance induced by optical irradiation in the $\mathrm{Fe} / \mathrm{SiO}_{2}$ / -Si hybrid structure with Schottky barrier. J Appl Phys. 114, 09 (2013)

24. Dezheng Yang, Fangcong Wang, Yang Ren Yalu Zuo, Yong Peng, Shiming Zhou, Desheng Xue. A Large Magnetoresistance Effect in $p-n$ Junction Devices by the Space-Charge Effect. Adv Funct Mater. 23, 2918-2923 (2013).

25. M. S. Sodha, A. Dixit and S. Srivastava, Applied Physics Letters, 94, 251501 (2009)

\section{Figures}




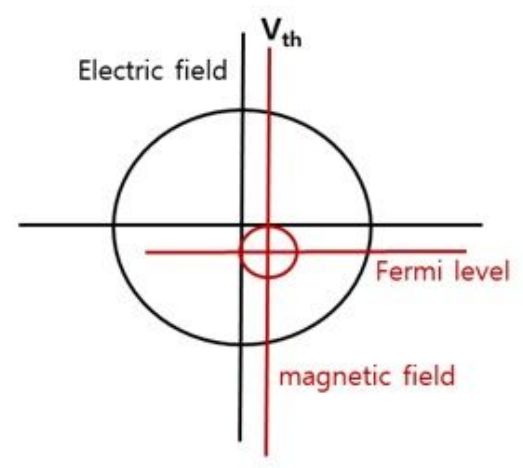

Law of Lenz

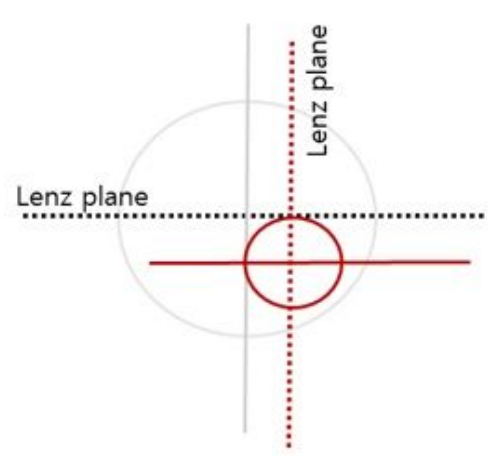

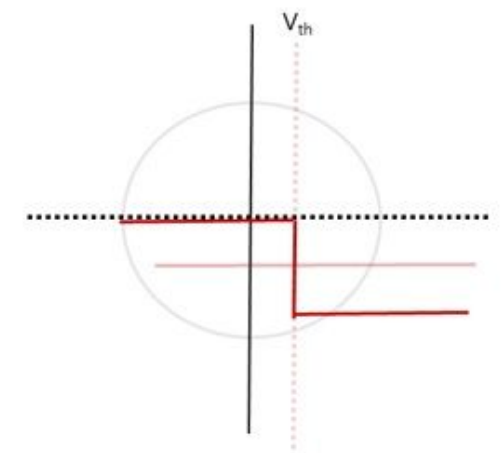

Dirac equation

Figure 1

Law of Lenz as the electromagnetic energy conservation and Lenz plane for Dirac equation.

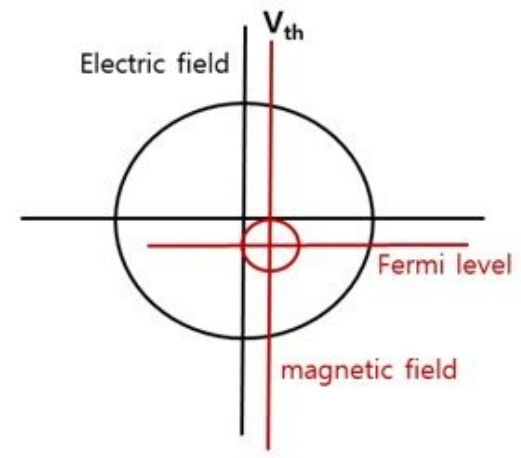

Law of Lenz

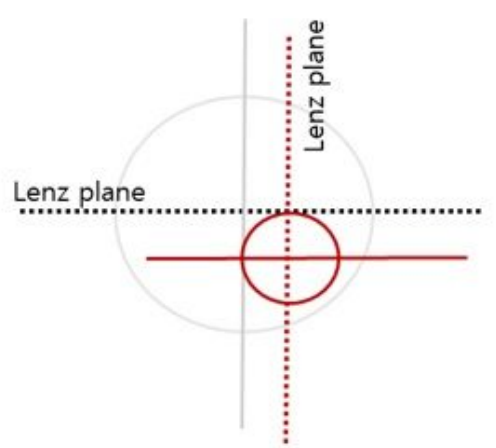

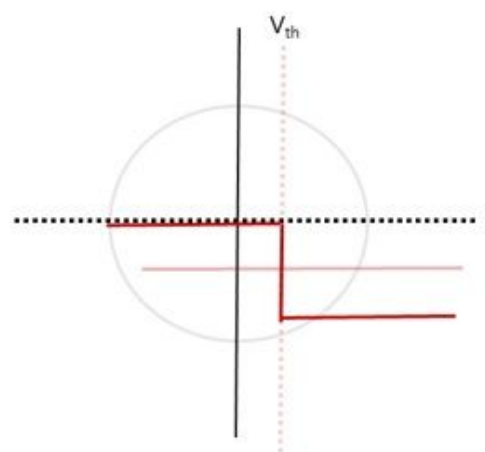

Dirac equation

\section{Figure 1}

Law of Lenz as the electromagnetic energy conservation and Lenz plane for Dirac equation.

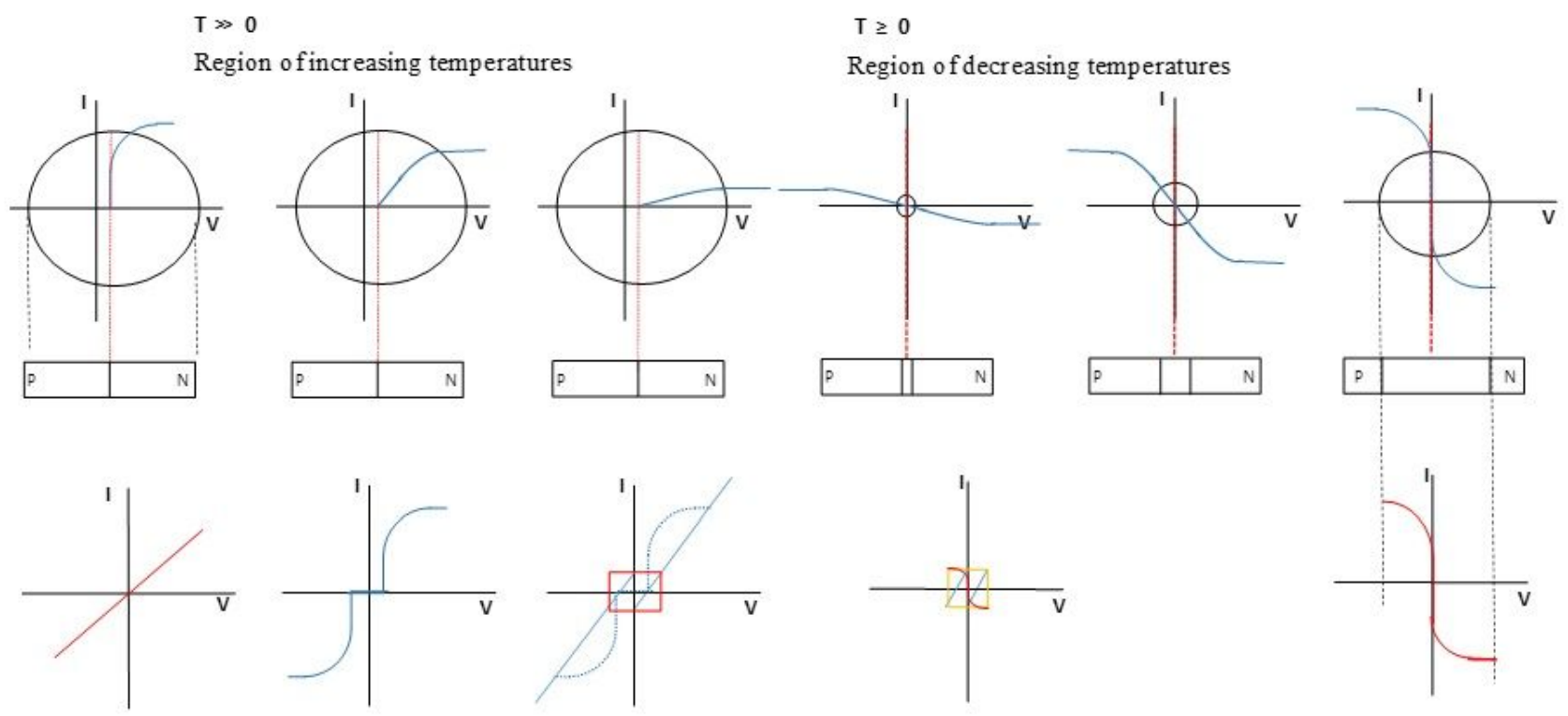


Figure 2

Difference between charge currents by electric fields in a PN junction and spin currents by magnetic fields in a schottky contact.

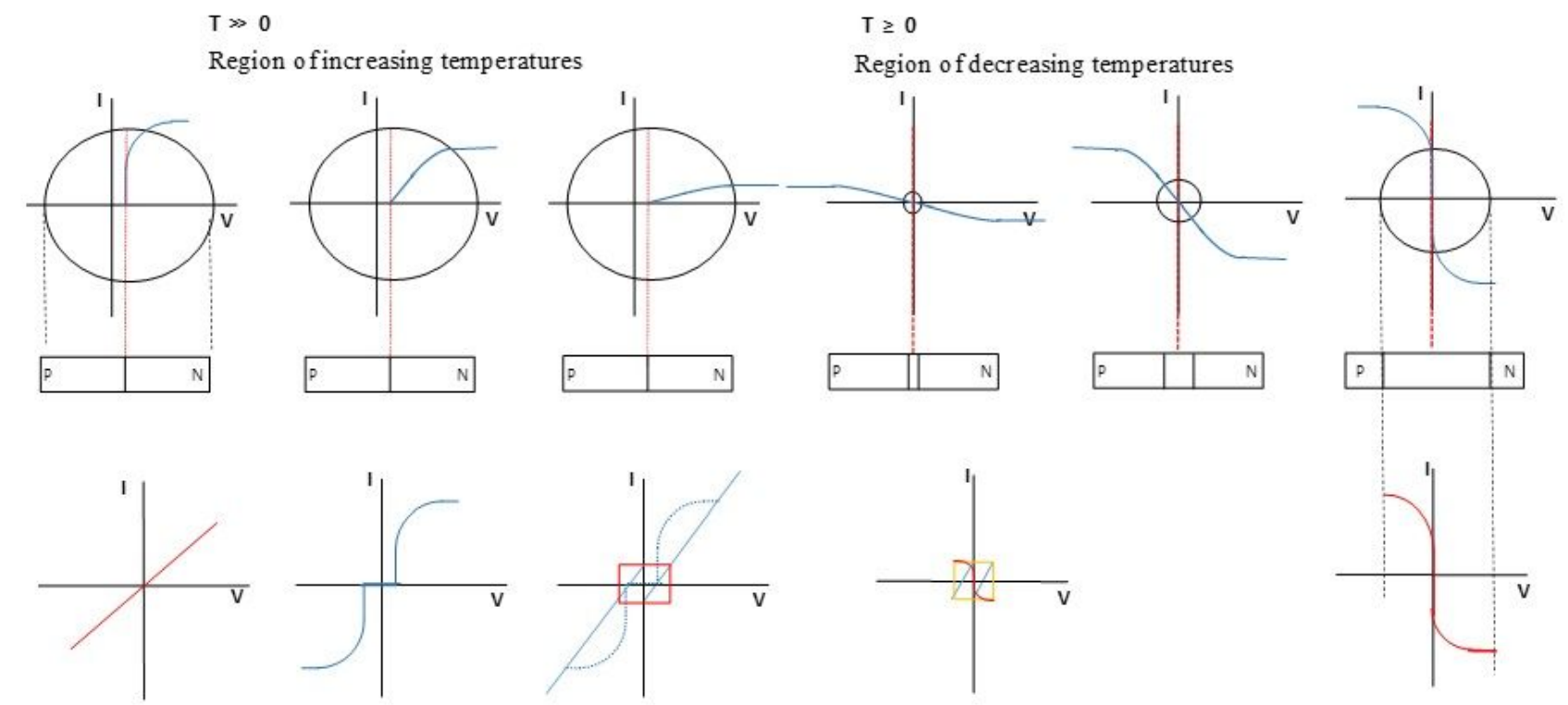

Figure 2

Difference between charge currents by electric fields in a PN junction and spin currents by magnetic fields in a schottky contact.
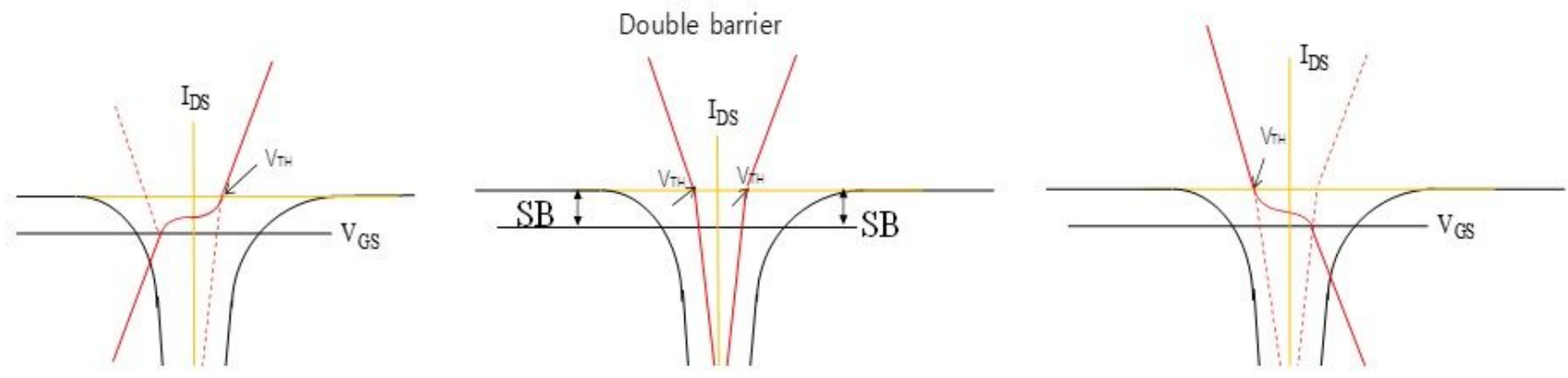

Figure 3

Stability of spin currents by double junction at Schottky contact.
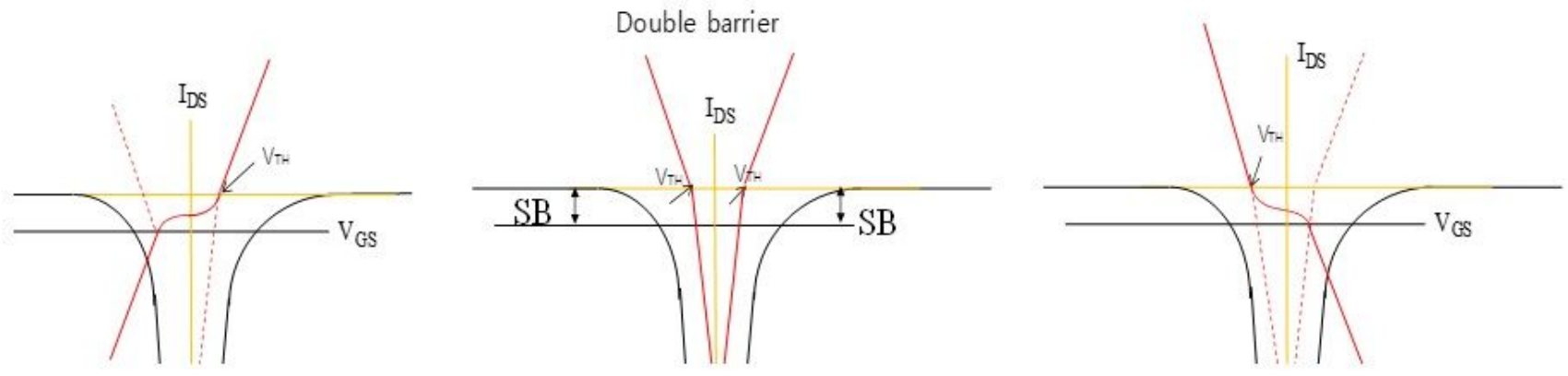

Figure 3

Stability of spin currents by double junction at Schottky contact. 

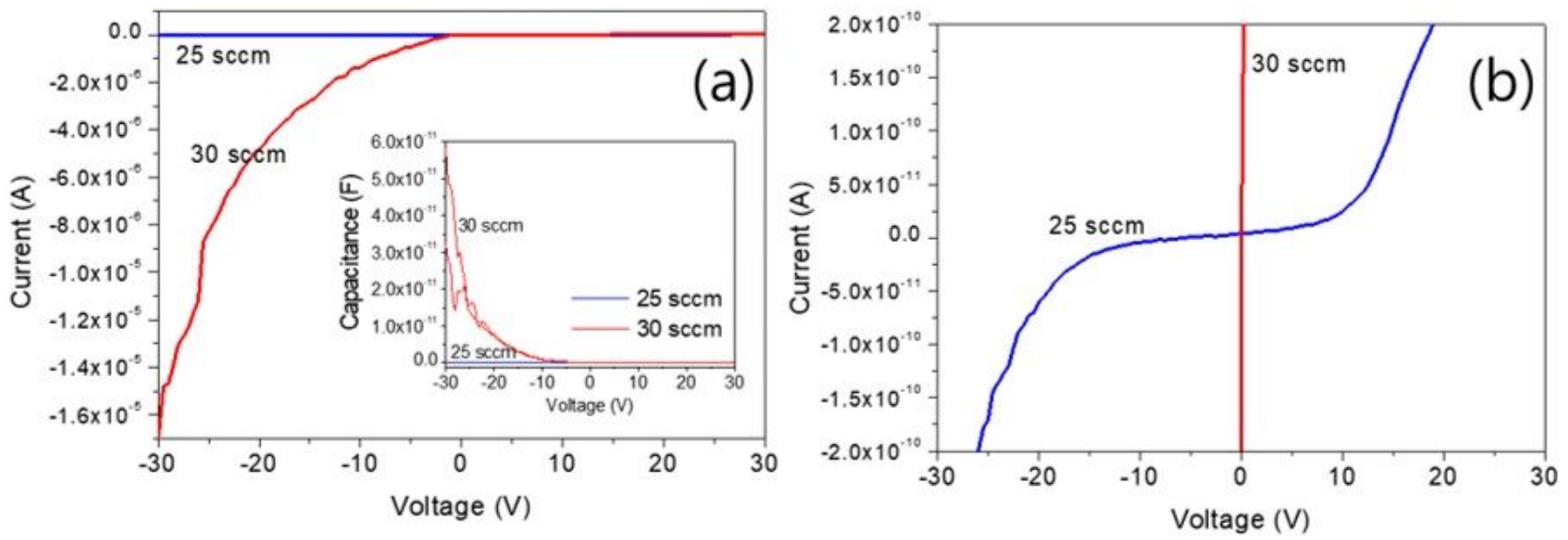

Figure 4

Electric properties of ZTO/SiO2 films with argon gas flow rates for deposited $\mathrm{SiO} 2,(\mathrm{a})$ current and capacitance, (b) current characteristics.
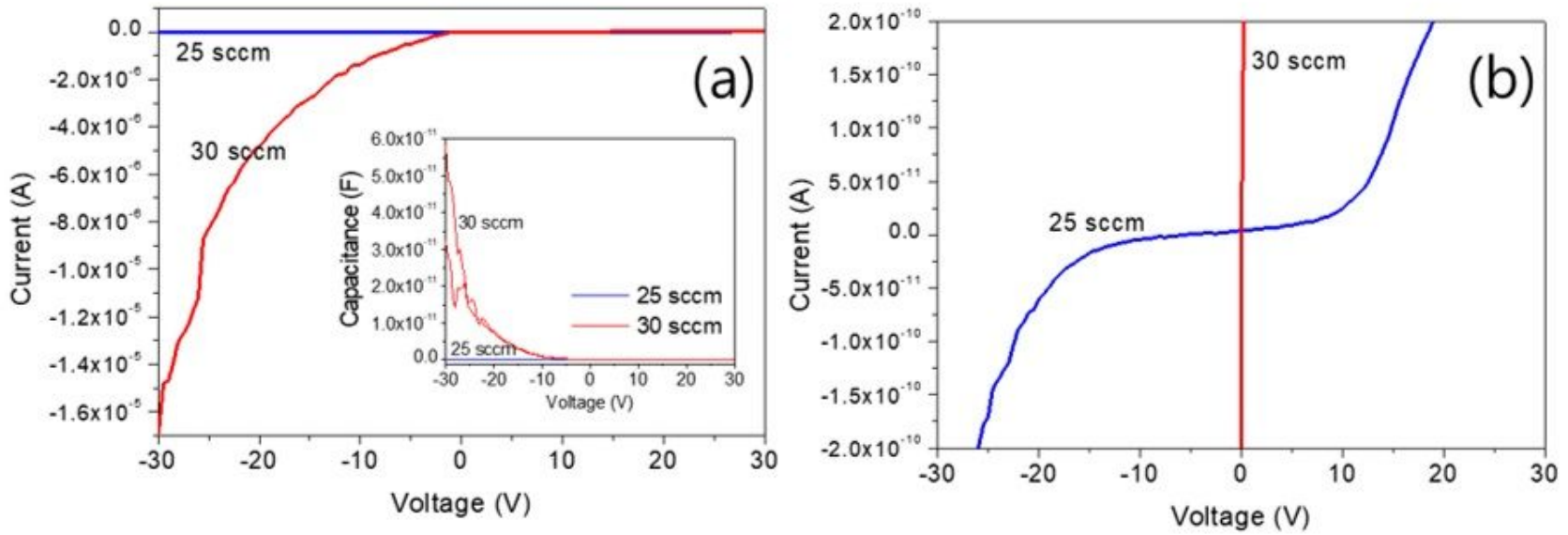

Figure 4

Electric properties of ZTO/SiO2 films with argon gas flow rates for deposited $\mathrm{SiO} 2$, (a) current and capacitance, (b) current characteristics.
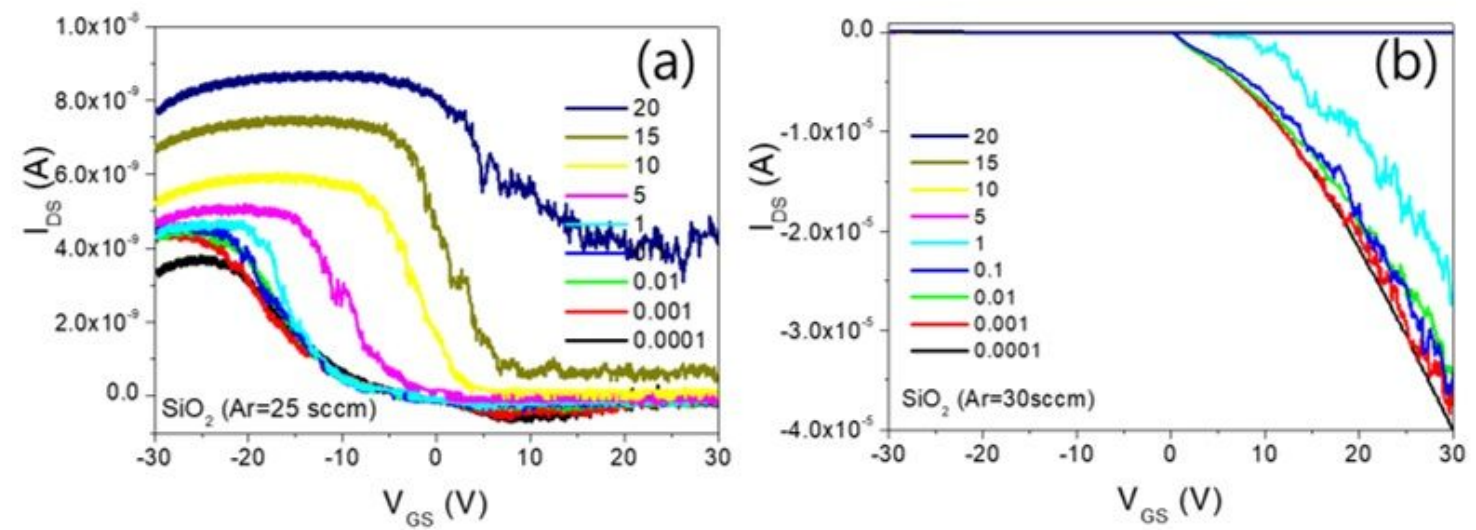

Figure 5

Transfer characteristics of ZTO/SiO2 transistor in according to the types of sin currents, (a) Weyl fermion, (b) Dirac fermion. 

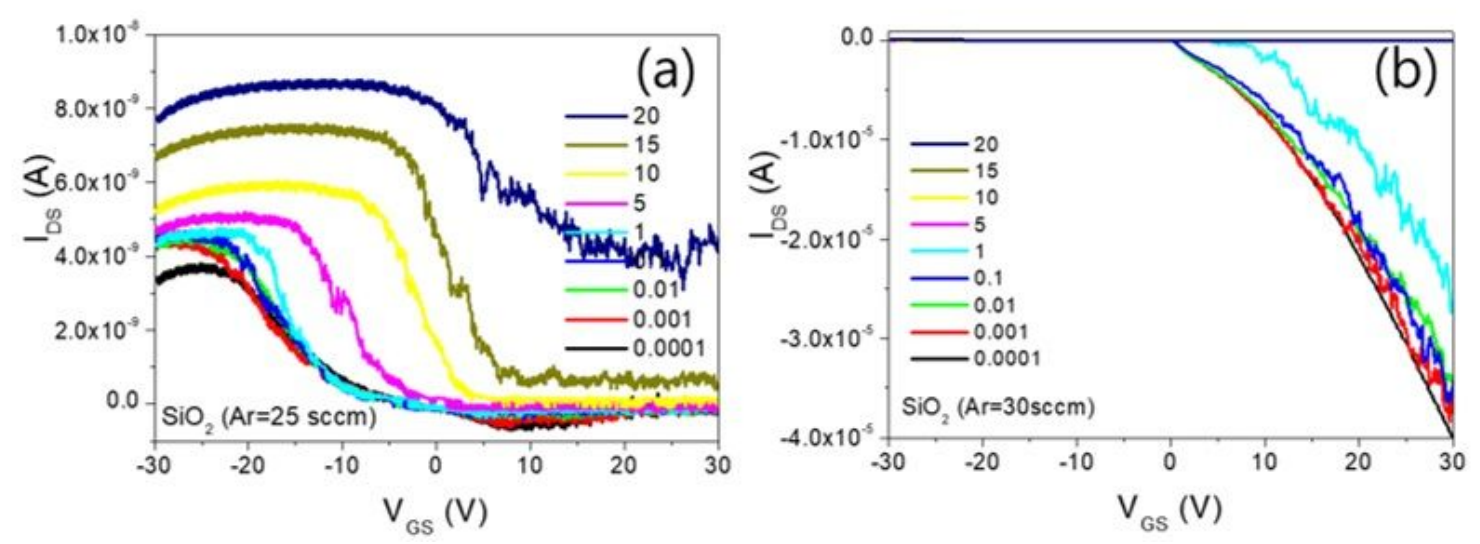

Figure 5

Transfer characteristics of ZTO/SiO2 transistor in according to the types of sin currents, (a) Weyl fermion, (b) Dirac fermion.

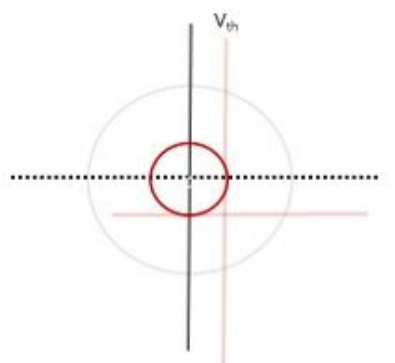

Majorana fermion Super current

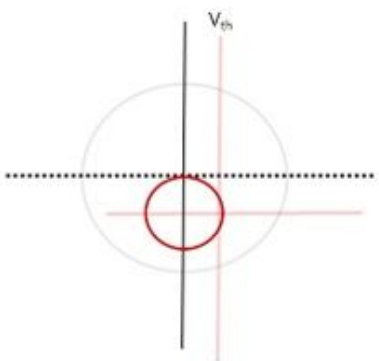

Dirac fermion Super current

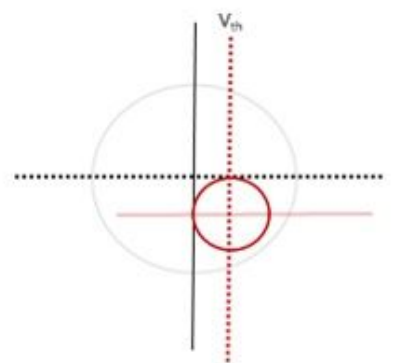

Dirac fermion

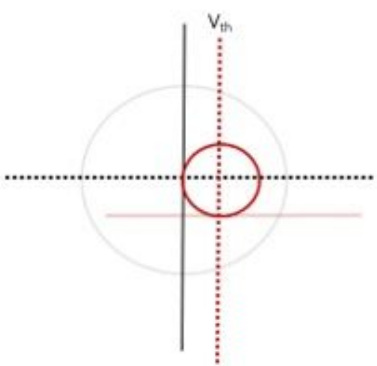

Weyl fermion

\section{Figure 6}

Majorana fermion, Dirac fermion and Weyl fermion in according to the Lenz plane.

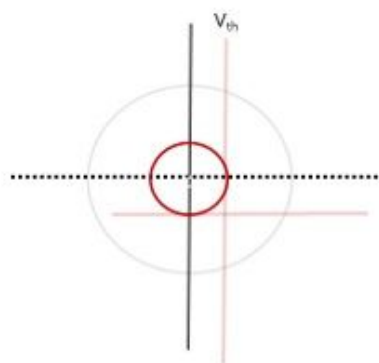

Majorana fermion Super current

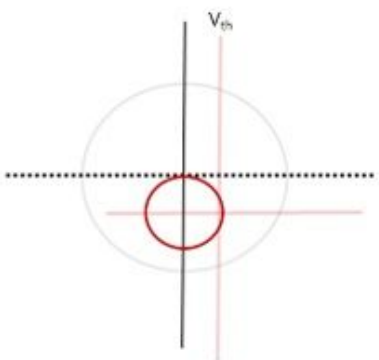

Dirac fermion Super current

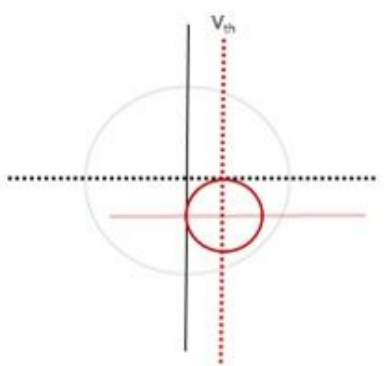

Dirac fermion

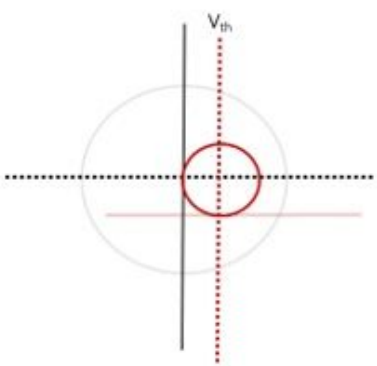

Weyl fermion

Figure 6

Majorana fermion, Dirac fermion and Weyl fermion in according to the Lenz plane. 

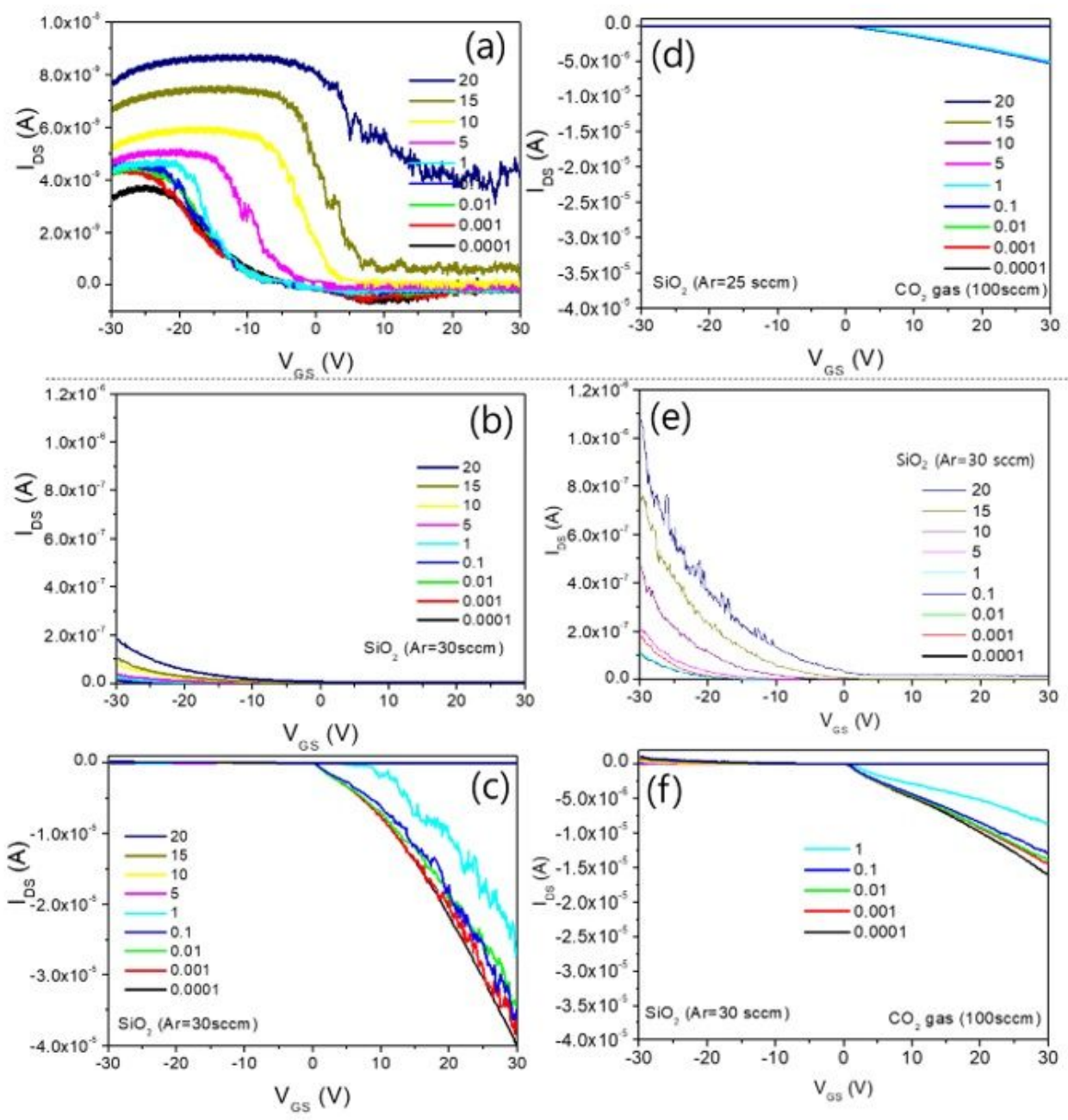

Figure 7

Responsibility of $\mathrm{CO} 2$ gas reaction of $100 \mathrm{sccm}$ between spin current and charge current, (a) Weyl fermion by $\mathrm{Ar}=25 \mathrm{sccm}$, (b) Dirac fermion by $\mathrm{Ar}=30 \mathrm{sccm}$, (c) Dirac fermion by Ar=30 sccm, transfer characteristics in $\mathrm{CO} 2$ gas environments; (d) negative current by Weyl fermion, (e) positive currents by Dirac fermion, (f) negative current by Dirac fermion. 

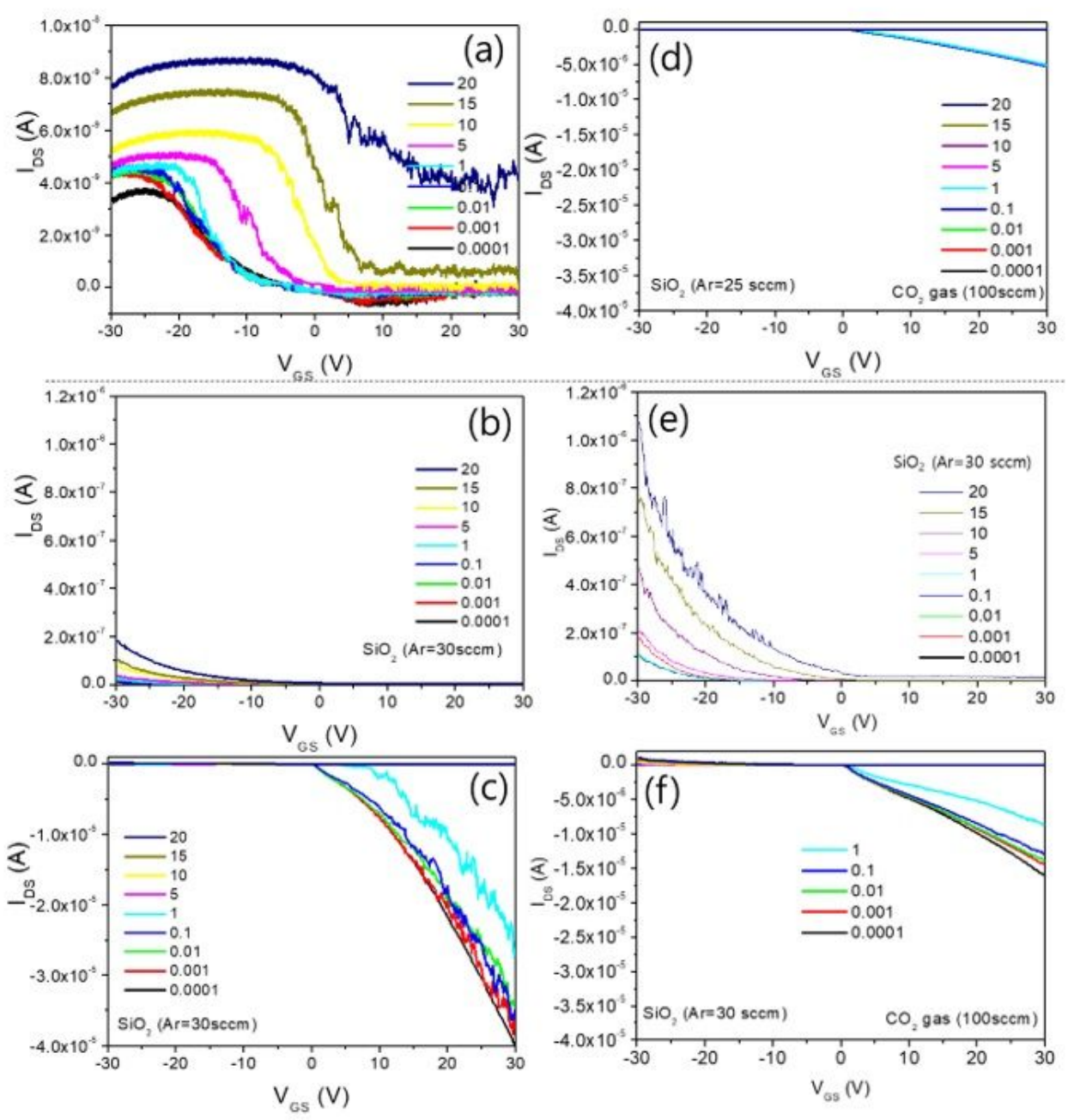

Figure 7

Responsibility of $\mathrm{CO} 2$ gas reaction of $100 \mathrm{sccm}$ between spin current and charge current, (a) Weyl fermion by $\mathrm{Ar}=25 \mathrm{sccm}$, (b) Dirac fermion by $\mathrm{Ar}=30 \mathrm{sccm}$, (c) Dirac fermion by Ar=30 sccm, transfer characteristics in $\mathrm{CO} 2$ gas environments; (d) negative current by Weyl fermion, (e) positive currents by Dirac fermion, (f) negative current by Dirac fermion.
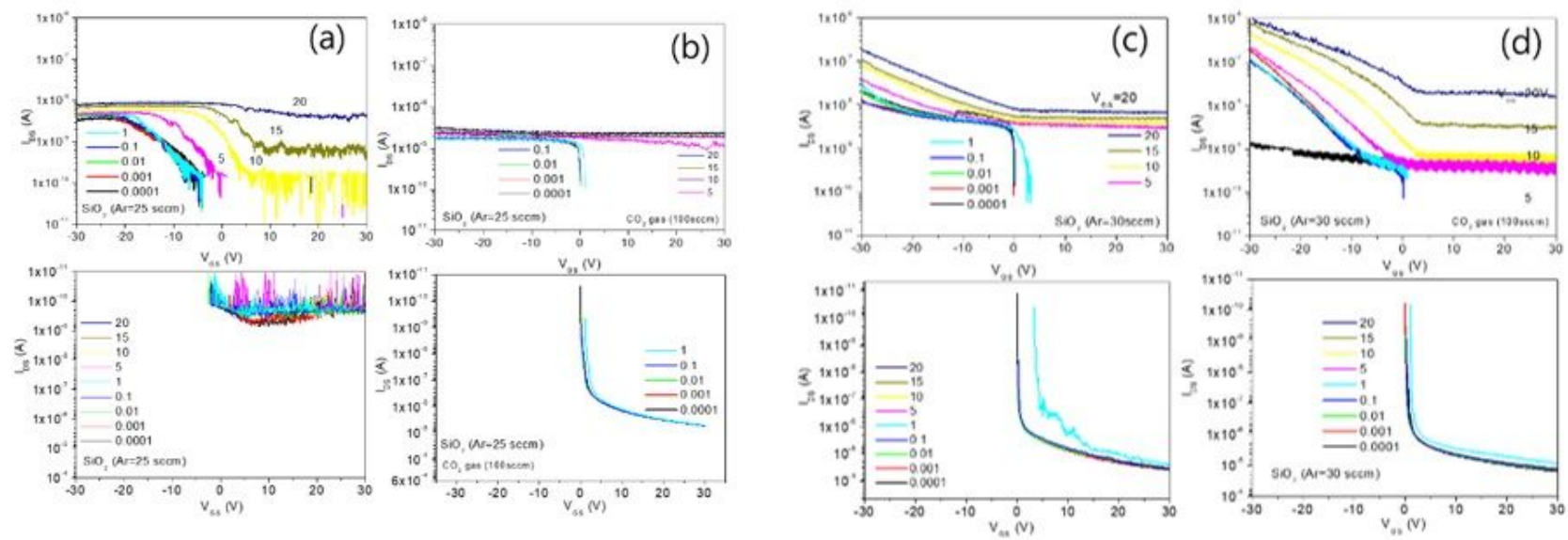

\section{Figure 8}

Log scale of transfer characteristics of TFTs between without CO2 gas and CO2 gas environments, (a) current by Weyl fermion, (b) effect of Weyl fermion in $\mathrm{CO} 2$ gas condition, (c) current by Dirac fermion, (d) effect of Dirac fermion in CO2 gas condition. 

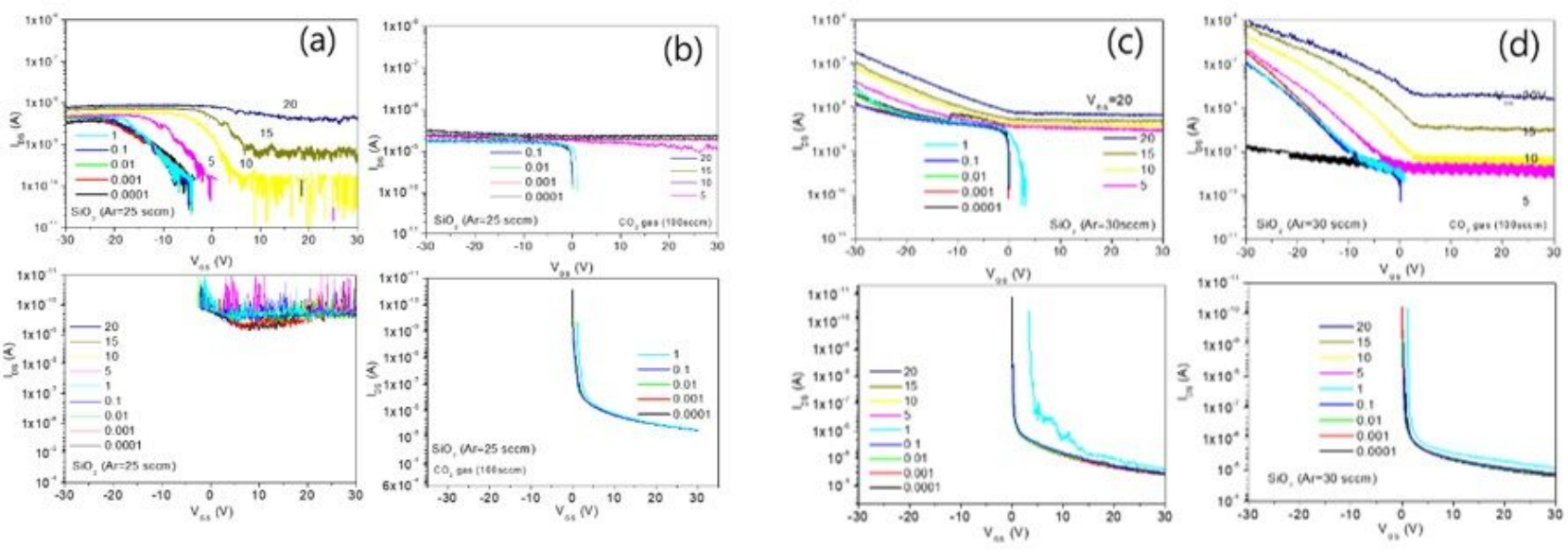

\section{Figure 8}

Log scale of transfer characteristics of TFTs between without $\mathrm{CO} 2$ gas and $\mathrm{CO} 2$ gas environments, (a) current by Weyl fermion, (b) effect of Weyl fermion in $\mathrm{CO} 2$ gas condition, (c) current by Dirac fermion, (d) effect of Dirac fermion in $\mathrm{CO} 2$ gas condition. 